\title{
Repercussões das abordagens mindful eating e intuitive eating na saúde de indivíduos adultos: revisão integrativa
}

\section{Repercussions of mindful eating and intuitive eating approaches on the health of adult individuals: an integrative review}

\section{Repercusiones de los enfoques de mindful eating e intuitive eating en la salud de individuos adultos: revisión integradora}

Recebido: $07 / 10 / 2020$
Aprovado: 11/05/2021
Publicado: $14 / 10 / 2021$

Ana Flávia de Sousa Silva 1 Camila Cremonezi Japur ${ }^{2}$ Taisa Alves Silva ${ }^{3}$ João Henrique Fabiano Motarelli ${ }^{4}$ Thamara Smatiotto Buttros 5 Fernanda Rodrigues de Oliveira Penaforte 6

Esta é uma revisão integrativa realizada no primeiro semestre de 2020, considerando o período de outubro de 2008 a dezembro de 2019 nas bases de dados PubMed, LILACS, SciELO, PsycINFO e Scopus, com o objetivo de conhecer a produção de pesquisas e as repercussões das abordagens centradas no "mindful eating" e no "intuitive eating" na saúde de indivíduos adultos. A questão norteadora foi: Quais as repercussões das abordagens centradas no "mindful eating" e no "intuitive eating" na saúde de indivíduos adultos? Para as buscas, foram utilizados descritores: mindful eating, intuitive eating e atenção plena. 0 corpus foi composto por 20 artigos na íntegra, todos internacionais, com três categorias: Resultados das abordagens centradas no mindful eating na saúde mental; Resultados das abordagens centradas no mindful eating na saúde física; e Resultados das abordagens centradas no intuitive eating na saúde mental e física. As intervenções baseadas em mindful eating e/ou intuitive eating repercutiram positivamente na saúde física e mental, melhorando o comportamento alimentar, as escolhas alimentares, reduzindo os níveis de alimentação emocional e estresse, auxiliando na perda de peso, na redução do índice de massa corporal e no tratamento de transtornos alimentares, sendo assim, estratégias promissoras para melhorar a saúde de indivíduos adultos.

Descritores: Saúde; Comportamento alimentar; Adulto.

This is an integrative review carried out in the first half of 2020, from October 2008 to December 2019 in the PubMed, LILACS, SciELO, PsycINFO and Scopus databases. It aimed to know the research production and repercussions of the approaches centered on "mindful eating" and "intuitive eating" in the health of adult individuals. The guiding question was: What are the repercussions of approaches centered on "mindful eating" and "intuitive eating" on the health of adult individuals? For the searches, descriptors were used: mindful eating, intuitive eating and "atenção plena" (mindfulness). The corpus consisted of 20 articles in full, all international, with three categories: Results of approaches centered on mindful eating in mental health; Results of approaches centered on mindful eating in physical health; and Results of intuitive eating-centered approaches to mental and physical health. Interventions based on mindful eating and/or intuitive eating had a positive impact on physical and mental health, improving eating behavior, food choices, reducing levels of emotional eating and stress, helping with weight loss, reducing body mass index and in the treatment of eating disorders, thus, promising strategies to improve the health of adult individuals.

Descriptors: Health; Feeding behavior; Adult.

Esta es una revisión integradora realizada en el primer semestre de 2020, considerando el periodo comprendido entre octubre de 2008 y diciembre de 2019 en las bases de datos PubMed, LILACS, SciELO, PsycINFO y Scopus, con el objetivo de conocer la producción de investigaciones y las repercusiones de los enfoques de "mindful eating" e "intuitive eating" en la salud de individuos adultos. La pregunta guía fue: ¿Cuáles son las repercusiones de los enfoques de "mindful eating" e "intuitive eating" en la salud de los adultos? Para las búsquedas se utilizaron descriptores: mindful eating, intuitive eating y atenção plena (atención plena). El corpus se compuso de 20 artículos completos, todos internacionales, con tres categorías: Resultados de los enfoques de mindful eating en la salud mental; Resultados de los enfoques de mindful eating en la salud física; y Resultados de los enfoques de intuitive eating en la salud mental y física. Las intervenciones basadas en mindful eating y/o intuitive eating han tenido repercusiones positivas en la salud física y mental, mejorando el comportamiento alimentario, la elección de alimentos, reduciendo los niveles de alimentación emocional y de estrés, ayudando a la pérdida de peso, reduciendo el índice de masa corporal y tratando los trastornos alimentarios, siendo por tanto estrategias prometedoras para mejorar la salud de individuos adultos.

Descriptores: Salud; Conducta alimentaria; Adulto.

1. Nutricionista. Mestre em Psicologia. Doutoranda em Nutrição e Metabolismo pela Universidade de São Paulo (USP), Campus Ribeirão Preto, SP, Brasil. 0RCID: 0000-0003-0000-8953 mail: anaflavia.s.s@usp.br

2. Nutricionista. Especialista em Avaliação Nutricional Intra-hospitalar. Mestre e Doutora em Ciências Médicas. Professora da Faculdade de Medicina de Ribeirão Preto (FMRP) e da Pós-Graduação em Nutrição e Metabolismo da USP. Ribeirão Preto, SP, Brasil. ORCID: 0000-0003-0513-1758 E-mail: camilajapur@usp.br

3. Nutricionista. Especialista em Saúde da Criança e do Adolescente. Mestranda em Nutrição e Metabolismo pela FMRP/USP. Ribeirão Preto, SP, Brasil. 0RCID: 0000-0001-7725-8470

mail: taisaalves@usp.br

4. Nutricionista. Profissional de Educação Física. Especialista em Mindfulness. Mestrando em Ciências Médicas e Biológicas pela Universidade Federal de São Paulo, SP, Brasil. ORCID: 0000-0002-5151-6950 E-mail: nutricionista@joaomotarelli.com

5. Nutricionista. Ribeirão Preto, SP, Brasil. ORCID: 0000-0002-3608-4424 E-mail: thamara.buttros@usp.br

6. Nutricionista. Especialista em Avaliação Nutricional Intra-Hospitalar. Mestre em Ciências Biomédicas. Doutora em Investigação Biomédica. Professora adjunta do curso de graduação em Nutrição e do Programa de Pós Graduação em Psicologia da Universidade Federal do Triângulo Mineiro. Uberaba, MG, Brasil. ORCID: 0000-0001-8483-1562 E-mail: fernandaropenaforte@gmail.com 


\section{INTRODUÇÃO}

A

tualmente, sabe-se que muitas doenças apresentadas pela população estão atreladas a hábitos de vida, especialmente os alimentares ${ }^{1}$. No entanto, os programas tradicionais de intervenção nutricional para auxiliar no tratamento dessas doenças não se mostram eficazes $^{2}$. Tal insucesso pode estar associado ao fato dos tratamentos convencionais se pautarem, quase que exclusivamente, na prescrição de dietas. Tal abordagem está centrada na restrição alimentar e na perspectiva biológica da alimentação, não abarcando a multidimensionalidade do comer e dos comportamentos relacionados à saúde 3 .

Nessa perspectiva, duas abordagens vêm ganhando destaque no cenário da alimentação e nutrição: o mindful eating e o intuitive eating. Mindful Eating (ME), ou sua tradução livre "atenção plena ao comer", consiste na habilidade de trazer uma atenção amorosa para todo o processo de comer, na qual os indivíduos estão atentos ao sabor dos alimentos, textura, sem julgamentos. A proposta é que haja atenção plena às sensações, sentimentos e pensamentos relacionadas à presença ou ausência da fome, tanto física quanto emocional ${ }^{4}$.

Assim, o ME é uma experiência que envolve corpo, mente e coração na escolha e preparo da comida, bem como no ato de comê-la ${ }^{4,5}$. Atualmente, existem diferentes programas para o manejo do comportamento alimentar por meio de programas de $\mathrm{ME}$, em diversos cenários clínicos. Esses programas são chamados de Intervenções baseadas em Mindfulness (Mindfulness Based Interventions-MBI), nos quais o ME é aplicado por meio dessas MBI's. Dentre estes, se destacam dois protocolos internacionalmente reconhecidos e que apresentam evidências: (1) Mindfulness Based Eating Awareness Training (MBEAT) ${ }^{6}$ e (2) Mindfulness Based Eating Solution (MBES-Eat for life) 7 .

Na mesma linha, o Intuitive Eating (IE) ou sua tradução livre "comer intuitivo", é uma abordagem que visa integrar a mente, o corpo e a comida, no qual o sujeito deve comer guiandose, principalmente, pela sua sinalização fisiológica de fome e saciedade. Para isso, é de extrema importância que haja uma conexão com os sinais internos do corpo. Os principais componentes do estilo de alimentação intuitivo são: permissão incondicional para comer; comer por razões físicas e não emocionais, e ter confiança no seu corpo e na sua sinalização de fome e saciedade para determinar quando e quanto comer8,9.

0 interesse nessas abordagens, relativamente novas, tem aumentado, mas a sistematização do conhecimento delas para a saúde de indivíduos adultos são poucos. Pode-se citar: verificar os efeitos do mindful eating na perda de peso ${ }^{10}$ e em comportamentos relacionados a obesidade e transtornos alimentares ${ }^{5}$. Outras pesquisas abordaram outros aspectos que não apenas os desfechos das intervenções com ME e IE na ingestão alimentar e saúde ${ }^{11}$, no comportamento alimentar ${ }^{12,13}$ e no peso ${ }^{14}$, mas também os efeitos de intervenções não-dieta e/ou baseadas na atenção plena, terapia de aceitação e compromisso e dialética comportamental.

São escassos os estudos que apresentassem somente resultados de intervenções baseadas no mindful eating e/ou no intuitive eating na saúde (notadamente os nacionais ${ }^{5}$ ), como um conceito multidimensional que envolve o bem-estar físico, mental e social proposto pela Organização Mundial da Saúde (OMS) ${ }^{15}$ e no qual essa revisão está amparada de indivíduos adultos.

A sistematização desse conhecimento é importante não só para a sua melhor compreensão, mas também para propagar, no Brasil, o conhecimento das pesquisas internacionais envolvendo essas abordagens (mindful eating e intuitive eating), contribuindo com a redução dessa lacuna do conhecimento. Desse modo, este estudo teve por objetivo conhecer a produção acerca de pesquisas e repercussões das abordagens centradas no "mindful eating" e no "intuitive eating" na saúde de indivíduos adultos. 


\section{MÉTODO}

Trata-se de uma revisão integrativa, que tem busca a síntese do estado de conhecimento acerca de um determinado assunto, discutindo-o de maneira integrada, na possibilidade de levantamento de lacunas que requerem novas pesquisas. 0 propósito inicial deste método é obter profundo entendimento de um determinado fenômeno baseando-se em estudos anteriores $^{16}$.

A revisão integrativa inclui a análise de pesquisas que são relevantes e que irão servir como base para a tomada de decisões e para a melhoria da prática clínica. 0 procedimento realizado nesta revisão seguiu as seguintes etapas: (1) identificação do tema e da questão norteadora; (2) estabelecimento de critérios de inclusão/exclusão; (3) categorização dos estudos; (4) avaliação dos estudos; (5) interpretação dos resultados e (6) síntese do conhecimento. Foi adotado também o protocolo internacional para estudos de revisão sistemática e metanálises, o PRISMA (Preferred Reporting Items for Systematic Reviews and Meta-Analyses), para orientar tanto a inclusão e exclusão dos artigos quanto a escrita da presente revisão ${ }^{16}$.

Para o presente trabalho a pergunta de pesquisa foi definida a partir da estratégia PICO, que prevê a definição do participante (P), intervenção (I), comparação (C) e desfecho/ outcomes (0), com exceção do item comparação que foi excluído da redação da pergunta, alteração que é prevista na metodologia PICO em alguns $\operatorname{casos}^{17}$. Pretende-se responder à questão norteadora: Quais as repercussões das abordagens centradas no "mindful eating" e no "intuitive eating" (I) na saúde $(0)$ de indivíduos adultos (P)?

A seleção dos artigos ocorreu em janeiro de 2020, e foi realizada por dois juízes independentes. Caso houvesse discordância entre os revisores quanto à adequação do estudo havia uma avaliação por um terceiro juiz. Foram realizadas buscas nas bases de dados PubMed, Literatura Latino-americana e do Caribe em Ciências da Saúde (LILACS), Scientific Eletronic Library Online (SciELO), PsycINFO e Scopus. Foram considerados trabalhos em português, inglês e espanhol. Estas bases de dados foram escolhidas por suas amplitudes, no âmbito nacional e internacional, publicada sobre o tema de interesse, e também por incluírem periódicos conceituados na área da saúde.

Por não haver descritores indexados segundo a padronização do DeCs/MeSh, para as buscas, foram utilizados descritores não indexados, mas que se relacionam diretamente ao tema, sendo eles: mindful eating, intuitive eating e atenção plena. Estes unitermos apareceram em, pelo menos, um dos seguintes campos de busca: título, resumo, assunto ou palavra-chave.

Os critérios de inclusão estabelecidos foram (a) artigos publicados entre outubro de 2008 e dezembro de 2019; (b) que abordassem diretamente a temática de interesse; (c) empíricos; (d) com adultos; (e) que estivessem disponíveis gratuitamente para leitura na íntegra; (f) que buscassem avaliar as repercussões das abordagens centradas no ME ou IE em algum desfecho de saúde; e (g) publicados nos idiomas português, inglês e/ou espanhol.

Foram excluídos: (a) estudos com crianças, adolescentes e idosos; (b) materiais como monografias, editoriais, livros, capítulos de livros, resenhas e resumos em anais de congressos; (c) estudos de associação; (d) artigos de revisão; e (e) estudos sobre elaboração e validação de protocolos ou de questionários. Os estudos que se repetiram em mais de uma base de dados foram computados apenas uma vez.

Para verificar se os artigos atendiam aos critérios de inclusão e exclusão, realizou-se uma avaliação por dois revisores independentes, obedecendo a seguinte ordem: (1) títulos de todos os estudos identificados; (2) resumos dos estudos selecionados na fase anterior e (3) leitura completa dos textos selecionados.

Após a exclusão dos artigos que não atenderam aos critérios de inclusão, foi realizado um fichamento de todos os artigos que compuseram o corpus de análise desse estudo, com as seguintes informações: título, autores, ano e local de publicação, base onde foi encontrado o 
estudo, delineamento, amostra, instrumentos utilizados, objetivos, principais resultados e principais conclusões.

Com posse dos artigos se construíram categorias com produções que guardassem similaridades entre si. 0 conceito de saúde da Organização Mundial da Saúde (OMS) ${ }^{15}$ foi usado para apoiar essa categorização. No âmbito do "bem-estar físico", foram consideradas questões relacionadas à própria saúde biológica ou qualquer comportamento que a afetasse diretamente. Portanto, foram consideradas as repercussões no bem-estar físico em relação ao peso, índice de massa corporal (IMC), na manutenção do peso, melhorias na glicemia de jejum, glicemia pós prandial, hemoglobina glicada e níveis de proteína $\mathrm{C}$ reativa.

Para o "bem-estar mental" foi considerada alguma repercussão que envolvesse a saúde psicológica como as escolhas alimentares, comportamento alimentar, alimentação emocional, consumo de doces em pacientes diabéticos, no tratamento de transtornos alimentares, sofrimento psicológico e apreciação corporal.

\section{RESULTADOS}

A combinação de descritores utilizados nas estratégias de busca em cada base de dados está descrita na Tabela 1. As buscas nas bases de dados originaram um total de 850 artigos. A base com maior número de artigos foi o PubMed (393), seguida pela PsycINFO (239), SCOPUS (200), LILACS (12) e SciELO (6).

Tabela 1. Referências encontradas nas bases de dados segundo a estratégia de busca utilizada. Ribeirão Preto, 2020.

\begin{tabular}{llc}
\hline Estratégia de Busca & Bases de dados & Número de artigos encontrados \\
\hline ((Mindful* OR intuitive) AND eating) & PsyclNFO & 239 \\
((Mindful* OR intuitive) AND eating) & PubMed & 393 \\
((Mindful* OR intuitive) AND eating) & SCOPUS & 200 \\
((Mindful* OR intuitive) AND eating) & LILACS & 12 \\
((Mindful* OR intuitive) AND eating) & SciELO & 6 \\
\hline Total & & $\mathbf{8 5 0}$ \\
\hline
\end{tabular}

Na primeira etapa da análise, excluíram-se 79 artigos por estarem duplicados e 50 artigos de revisões da literatura. Posteriormente, foram excluídos 628 artigos, principalmente por não abordarem diretamente a temática de interesse. Restaram 93 artigos para leitura na íntegra e, após essa leitura, 73 artigos foram excluídos também por não estarem adequados aos critérios de inclusão, totalizando 20 artigos que compuseram o corpus dessa revisão. A Figura 1 apresenta o fluxograma de estratégia de seleção dos estudos de acordo com as normas PRISMA. 
Figura 1. Etapas de seleção dos artigos incluídos na revisão integrativa. Ribeirão Preto, 2020.

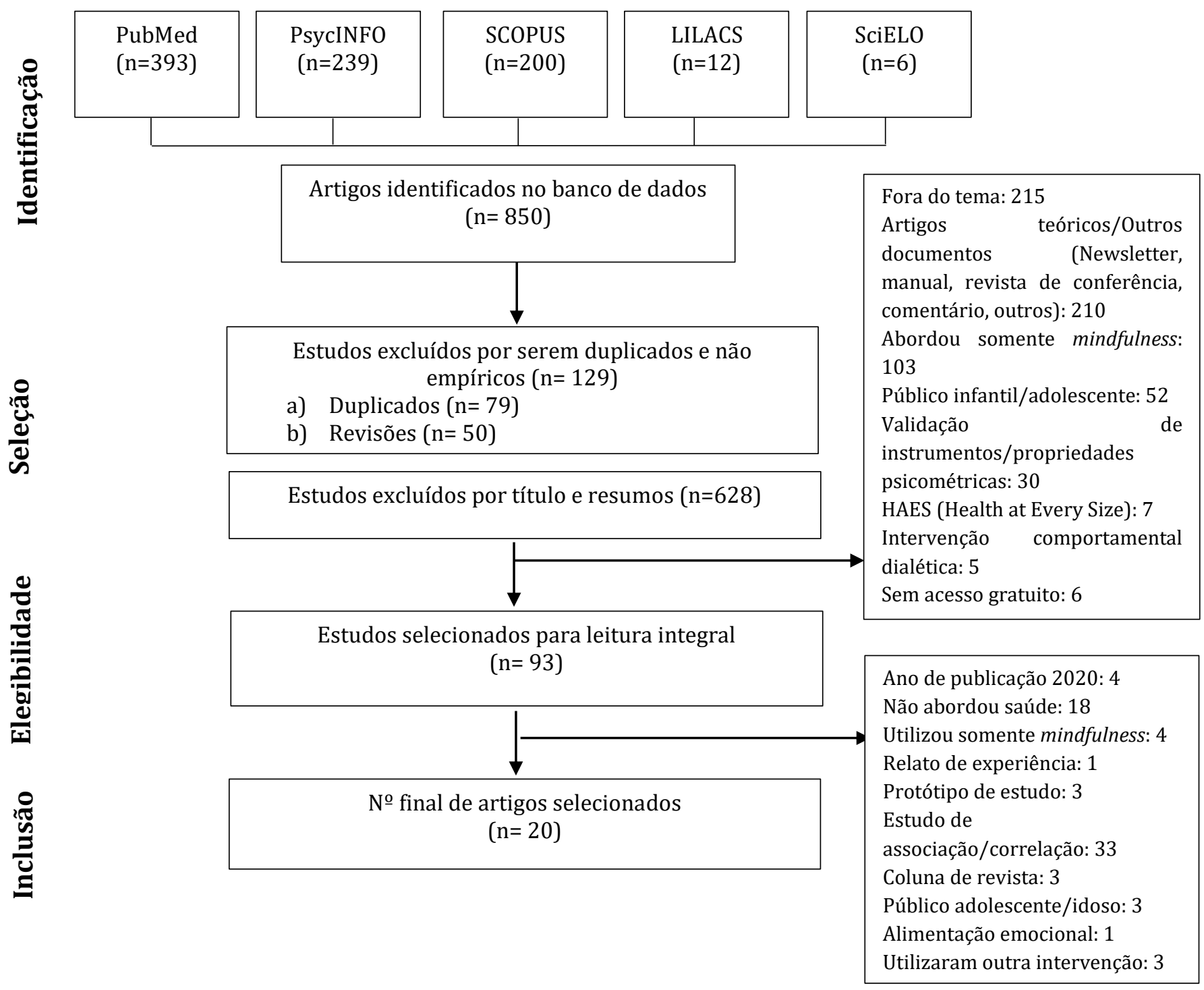

A Tabela 2 mostra informações relacionadas à autoria, o ano de publicação, amostra, delineamento, objetivo central e resultados principais encontrados nos artigos selecionados.

Os artigos que compõem a presente revisão são exclusivamente internacionais e no idioma inglês. 0 país com maior número de publicações foram os Estados Unidos ( $\mathrm{n}=14$; $70,0 \%)$, seguido por Austrália $(n=03 ; 15,0 \%)$, Canadá $(n=01 ; 5,0 \%)$, Espanha $(n=01 ; 5,0 \%)$ e Tailândia $(n=01 ; 5,0 \%)$. 0 ano com maior quantidade de publicação foi $2010(n=04 ; 20,0 \%)$, seguido por 2019, 2017, 2014 e 2012 com 3 artigos (15,0\%) em cada ano. Os anos de 2018, 2016, 2015, e 2013 foram os com menor número de publicações, contando com apenas 01 artigo $(5,0 \%)$ em cada ano.

0 tamanho amostral dos estudos variou de 01 (menor número amostral) ${ }^{34}$ a 194 (maior número amostral) ${ }^{25}$ de participantes. A maior parte dos estudos foi desenvolvida com homens e mulheres $(n=10 ; 50,0 \%)$, contudo uma parcela importante dos estudos foi realizada exclusivamente com mulheres $(n=09 ; 45,0 \%)$ e apenas $01(5,0 \%)$ foi realizado exclusivamente com homens.

Grande parte dos estudos foi realizada com sujeitos com sobrepeso e obesidade ( $\mathrm{n}=06$; $30,0 \%)$, seguido por portadores de transtornos alimentares $(n=03 ; 15,0 \%)$ e indivíduos com diabetes $(n=02 ; 10,0 \%)$. Foi encontrado $01(5,0 \%)$ estudo feito com gestantes com diabetes mellitus gestacional e 01 (5,0\%) estudo realizado com sujeitos com doença mental grave. 
No que tange ao tipo do estudo, a maioria dos artigos se enquadra no tipo randomizado ( $n=10 ; 50,0 \%)$, seguido pela avaliação dos participantes antes e após a intervenção (n= 07; $35,0 \%)$. A intervenção mais longa durou 5,5 meses $^{24}$, e a mais curta teve duração de uma única sessão $^{23}$. 0 tempo de intervenção mais comumente encontrado nos estudos foi de 8 semanas $(n=03,15,0 \%)$ e 6 semanas $(n=03,15,0 \%)$. A maioria dos estudos utilizou a intervenção baseada no mindful eating $(\mathrm{n}=16 ; 80,0 \%)$, seguida pelas intervenções que utilizaram o intuitive eating $(n=04 ; 20,0 \%)$.

É importante mencionar também que as pesquisas com grupo controle $(n=12 ; 60,0 \%)$ contaram com grupo controle ativo em 06 estudos (30\%), ou seja, os participantes receberam algum tipo de intervenção que não envolvia mindful eating e intuitive eating e 06 estudos $(30,0 \%)$ não fizeram nenhum tipo de intervenção no grupo controle.

Em relação ao uso de protocolos padronizados observou-se que em 65,0\% dos estudos $(n=13)$ foram utilizados grupos com intervenções baseadas em protocolos internacionalmente reconhecidos. Daqueles que não utilizaram, houveram estratégias como o uso de aplicativos ou recursos online para algumas sessões de mindful eating ou intuitive eating $(\mathrm{n}=04 ; 20 \%)$. Em $10,0 \%(n=02)$ dos estudos foi feita a combinação com métodos de mindfulness e o outro foi realizado em sessão individualizada $(n=01 ; 5,0 \%)$. Dentre os estudos analisados, a condução das intervenções feita por intermédio de um profissional capacitado e habilitado em ME ou IE foi citado em 55,0\% (n=11) dos artigos. 
Tabela 2. Estudos envolvendo intervenções baseadas no mindful eating ou no intuitive eating. Ribeirão Preto, 2020.

\begin{tabular}{|c|c|c|}
\hline Autoria e local & Amostra & Objetivo principal e delineamento \\
\hline $\begin{array}{l}\text { Lyzwinski et al. } \\
\text { (2019) }^{18} \\
\text { Australia }\end{array}$ & $\begin{array}{l}90 \text { estudantes } \\
\text { universitários } \quad(45 \\
\text { alocados no grupo } \\
\text { intervenção ME e } 45 \text { no } \\
\text { grupo controle). }\end{array}$ & $\begin{array}{l}\text { Testar a eficácia, a aceitabilidade e a viabilidade de um } \\
\text { aplicativo que combinava técnicas de redução de estresse } \\
\text { baseadas em mindfulness e ME no peso, comportamento } \\
\text { alimentar e níveis de estresse. } \\
\text { Duração da intervenção: } 11 \text { semanas. } \\
\text { Estudo controlado randomizado. }\end{array}$ \\
\hline $\begin{array}{l}\text { Gidugu } \\
\text { Jacobs } \\
(2019)^{19} \\
\text { Estados Unidos }\end{array}$ & $\begin{array}{l}46 \text { indivíduos com } \\
\text { doença mental grave. }\end{array}$ & $\begin{array}{l}\text { Avaliar o efeito de um programa baseado em ME e } \\
\text { educação alimentar no comportamento de saúde em } \\
\text { relação à alimentação em indivíduos com doença mental } \\
\text { grave. } \\
\text { Duração da intervenção: } 14 \text { semanas. } \\
\text { Delineamento experimental de corte longitudinal. Estudo } \\
\text { com avaliação do tipo antes e após a intervenção. }\end{array}$ \\
\hline $\begin{array}{l}\text { Dibb-Smith et } \\
\text { al. } \\
(2019)^{20} \\
\text { Austrália }\end{array}$ & 158 indivíduos & $\begin{array}{l}\text { Investigar os efeitos de um exercício de ME enviado por e- } \\
\text { mail (adaptado da "meditação da uva-passa"), a ser } \\
\text { realizado com alimentos escolhidos pelos participantes, } \\
\text { nos hábitos indesejados de "beliscar" alimentos (consumo } \\
\text { frequente de alimentos entre as refeições), níveis de ME e } \\
\text { de autocompaixão. } \\
\text { Duração da intervenção: } 2 \text { semanas. } \\
\text { Delineamento experimental de corte longitudinal. Estudo } \\
\text { com avaliação do tipo antes e após a intervenção. }\end{array}$ \\
\hline $\begin{array}{l}\text { Webber et al. } \\
(2018)^{21} \\
\text { Estados Unidos }\end{array}$ & $\begin{array}{l}26 \text { indivíduos }(14 \\
\text { alocados no grupo } \\
\text { intervenção IE e } 12 \text { no } \\
\text { grupo controle EBT- } \\
\text { programa redução de } \\
\text { estresse) }\end{array}$ & $\begin{array}{l}\text { Comparar os efeitos das duas abordagens (IE e EBT- } \\
\text { programa redução de estresse) no peso, comportamento } \\
\text { alimentar e níveis de estresse e depressão. } \\
\text { Duração da intervenção: } 14 \text { semanas. } \\
\text { Estudo controlado randomizado. }\end{array}$ \\
\hline $\begin{array}{l}\text { Wnuk et al. } \\
(2017)^{22} \\
\text { Canadá }\end{array}$ & 22 mulheres adultas & $\begin{array}{l}\text { Avaliar os efeitos de uma intervenção baseada no MB-EAT } \\
\text { na manutenção do peso e em sintomas psicológicos após a } \\
\text { cirurgia bariátrica. } \\
\text { Duração da intervenção: } 16 \text { semanas } \\
\text { Delineamento experimental de corte longitudinal. } \\
\text { Estudo com avaliação do tipo antes e após a intervenção. }\end{array}$ \\
\hline
\end{tabular}

\begin{abstract}
Ao final da intervenção, não houve diferenças em relação ao peso entre os grupos experimental vs. controle. O grupo intervenção ME apresentou menores níveis de estresse, de alimentação emocional e de alimentação descontrolada, além de maiores níveis de mindfulness e de ME vs. grupo controle.
\end{abstract}

Após a intervenção os participantes diminuiram os escores de comer emocional e de comer excessivo, e aumentaram os escores de comer consciente. Houve aumento nas pontuações relacionadas ao hábito
indesejado de "beliscar" e redução nos níveis de autocompaixão após a intervenção, sem alteração para os níveis de ME.

Não houve diferença para a perda de peso, comportamento alimentar e parâmetros emocionais entre os grupos; bem como intra-grupos ao final das intervenções.

Após a intervenção, houve redução significativa nos níveis de depressão, alimentação emocional e compulsão alimentar; e manutenção do peso entre as participantes. 
Allirot et al. $\quad 70$ mulheres adultas (2017) ${ }^{23} \quad$ (35 alocadas no grupo Espanha intervenção ME e 35 no grupo controle).

\section{Mason et al} $(2017)^{24}$

104 mulheres sobrepeso

Estados Unidos obesidade.

Mason et al.

(2016) $^{25}$

Estados Unidos

194 indivíduos com obesidade $(100$ do grupo intervenção baseada em mindfulness e ME e 94 do grupo controle, que recebeu informações gerais sobre nutrição e atividade física).

Stites et al. 26 adultos com

$(2015)^{26}$

sobrepeso

ou

Estados Unidos obesidade (10 alocados no grupo intervenção ME 8 semanas e 16 no grupo "intervenção curta" de 4 semanas).

Youngwanicha et al. (2014) ${ }^{27}$

Tailândia diabetes mellitus

\section{0 gestantes com} gestacional (DMG) (85 alocadas no grupo intervenção ME e 85 no
Avaliar o efeito de uma abordagem centrada no ME nas escolhas alimentares, ingestão alimentar, paladar e comportamentos alimentares externos (comer em resposta a sinais externos: cheiro ou aparência do alimento, independente dos sinais internos de fome e saciedade) e emocionais em mulheres adultas.

Duração da intervenção: Sessão única.

Delineamento experimental de corte tranversal, com avaliação após intervenção.

via smartphone, na compulsão alimentar, desejo alimentar, alimentação emocional e fome física.

Duração da intervenção: 12 semanas.

Delineamento experimental de corte longitudinal. Estudo com avaliação tipo antes e após a intervenção.

Avaliar os efeitos de uma intervenção baseada mindfulness e ME no consumo de doces e nos níveis de glicemia de jejum.

Duração da intervenção: 5,5 meses.

Estudo controlado randomizado

Avaliar o efeito de uma intervenção baseada em ME na promoção de compras de almoços mais saudáveis (meno teor de kcal e de gorduras).

Duração da intervenção: 8 semanas grupo intervenção

versus 4 semanas no grupo "intervenção curta".

Estudo controlado randomizado.

Investigar o efeito de uma intervenção baseada em ME e om prática de yoga nos níveis glicêmicos das gestantes com DMG

Duração da intervenção: 8 semanas

Estudo controlado randomizado de corte longitudinal.
A abordagem do ME melhorou as escolhas alimentares e a ingestão alimentar das participantes, reduzindo significativamente os comportamentos alimentares por razões externas e o consumo de alimentos com alta densidade calórica vs. grupo controle. Não houve efeito sobre o paladar dos indivíduos.

A intervenção baseada no ME gerou reduções significativas nos escores de compulsão alimentar, alimentação emocional e de fome física. Houve também redução no peso das participantes.

A intervenção baseada em mindfulness e ME gerou redução significativa no consumo de doces e na glicemia de jejum quando comparada ao grupo controle.

Os participantes do grupo intervenção ME compraram almoços com menor teor calórico e de gorduras em comparação com o grupo "intervenção curta".

0 grupo com intervenção baseada em ME apresentou redução significativa da glicemia de jejum, glicemia pós prandial (2 horas) e hemoglobina glicada, quando comparada ao grupo controle. 
grupo controle, sem

nenhuma intervenção).

Bush et al. 124 mulheres (53

(2014) $^{28}$ alocadas no grupo

Estados Unidos intervenção e 71 no grupo controle - lista de espera).

Miller et al. $\quad 52$ indivíduos $\quad(27$

(2014) $^{29}$ alocados no grupo

Estados Unidos Treinamento de Consciência Alimentar Baseado

em

Mindfulness adaptado para diabetes (MBEAT

D); e 25 no grupo Smart Choices - SC)

Kidd et al.

(2013) $^{30}$ 12 mulheres obesidade

\section{Estados Unidos}

Anglin

(2012) $^{31}$

Estados Unidos no grupo intervenção

com IE e 8 no grupo

$\begin{array}{lr}\text { com IE e } 8 & \text { no grupo } \\ \text { controle } & \text { com }\end{array}$

intervenção de

restrição calórica).

Timmerman e 35 mulheres (19 Avaliar o efeito da abordagem ME no controle de peso em

Brown

$(2012)^{32}$ alocadas no grupo mulheres que comiam em restaurante pelo menos 3 vezes intervenção com ME e por semana.

Estados Unidos 16 no grupo controle, Duração da intervenção: 6 semanas.

sem nenhuma Estudo controlado randomizado.

Examinar a eficácia de uma intervenção baseada no IE e ME (Protocolo Eat for Life) no comportamnto alimentar apreciação corporal, níveis de IE e de atenção plena. Duração da intervenção: 10 semanas.

Delineamento experimental de corte longitudinal

Avaliar o impacto de uma intervenção baseada na educação a protocolo MBEAT-D na ingestão alimentar, diabetes, níveis de atenção plena e sintomas depressivos. Duração da intervenção: 3 meses.

Estudo prospectivo randomizado controlado

Descrever os efeitos de uma intervenção baseada no ME nos níveis de alimentação consciente e de depressão; no peso, gordura corporal e pressão arterial; e na autoeficácia para perda de peso.

Duração da intervenção: 8 semanas.

Delineamento experimental.

Estudo com avaliação do tipo antes e após a intervenção.

Avaliar os efeitos de uma abordagem centrada no IE vs. peso, valores de IMC e de circunferência da cintura (CC).

Duração da intervenção: 6 semanas.

Estudo controlado randomizado.

intervenção).
O grupo intervenção apresentou maiores pontuações para apreciação corporal, níveis de IE e de atenção plena; e maiores chances de serem assintomáticos para transtornos alimentares $v s$. grupo controle.

O grupo SC teve maior aumento no conhecimento e crenças eficazes na autogestão do diabetes vs. MBEAT-D, após intervenção. Para o consumo de frutas e legumes, níveis de atenção plena, sintomas depressivos e conhecimento e autoeficácia relacionados à nutrição e alimentação não houve diferença entre os grupos.

A intervenção de ME aumentou a autoeficácia para perda de peso. Não houve em mudanças significativas para os níveis de alimentação consciente e de depressão; e para o peso, gordura corporal e pressão arterial após a intervenção.

A perda de peso e a redução nos valores de IMC foram significativamente maiores no grupo RC vs. IE após intervenção. Para a CC não houve diferenças entre os grupos.

As participantes do grupo ME perderam mais peso, reduziram a ingestão calórica e a ingestão de gordura quando comparado ao grupo controle. 
Miller et al. (2012) $^{33}$ Estados Unidos

52 indivíduos alocados no grup

Treinamento

de

Consciência Alimentar

Baseado

em

Mindfulness adaptado

para diabetes (MBEAT-

D); e 25 no grupo Smart

Choices - SC).

Albers

$(2010)^{34}$

1 estudante

Estados Unidos

Cole e

Horacek

(2010) $^{35}$

Estados Unidos

32 mulheres de uma

instalação militar (18

alocadas no grupo

intervenção IE e 14 no

grupo controle, sem

nenhuma intervenção)

Hepworth

(2010) ${ }^{36}$

33 mulheres

com

Austrália

Dalen et al.

(2010) $^{37}$

transtornos

alimentares.

Estados Unidos
Estudo com avaliação do tipo antes e após a intervenção.

10 indivíduos com Verificar o impacto de um programa baseado em ME obesidade. (MEAL) no peso, IMC, comportamento alimentar, saúde mental e marcadores fisiológicos (risco cardiovascular como: glicose, LDL, adiponectina).

Duração da intervenção: 6 semanas.

Delineamento experimental de corte longitudinal. Estudo com avaliação do tipo antes e após a intervenção.
Ambos os grupos apresentaram diminuição de peso, IMC, CC níveis de hemoglobina glicada e ingestão energética no final das intervenções, sem diferença entre eles. Entre os grupos, houve diferença para o consumo de gorduras trans (menor consumo no grupo SC), de fibra total (maior consumo no grupo SC) e de açúcares totais (menor consumo no grupo MBEAT-D), ao final da intervenção.

Após a abordagem baseada no ME, houve redução da restrição alimentar e aumento do IMC e da ingestão calórica. Houve também melhora em relação ao sofrimento emocional.

Duração da intervenção: 15 sessões (que foram realizadas

melhoria da mentalidade da dieta.

Duração da intervenção: 10 semanas.

Investigar os benefícios de um grupo baseado em ME no transtornos alimentares.

Duração da intervenção: 10 semanas

Delineamento exploratório de corte longitudinal.

O programa melhorou a mentalidade da dieta das participantes, que iniciaram comportamentos de vida mais intuitivos, reduziram a alimentação emocional e aumentou a autoestima das mesmas vs. grupo controle.

Houve melhora significativa nos escores do EAT-26 (usado para avaliar sintomas e comportamentos característicos de transtornos alimentares) após a participação no grupo baseado em ME.

Após o programa, foi observada redução significativa no peso e nos níveis de proteína $\mathrm{C}$ reativa, melhoria nos escores de compulsão alimentar e de sofrimento psicológico. 
Os resultados foram organizados em 3 categorias: Resultados das abordagens centradas no mindful eating na saúde mental (13 artigos); Resultados das abordagens centradas no mindful eating na saúde física (10 artigos); e Resultados das abordagens centradas no intuitive eating na saúde mental e física (4 artigos). Algumas produções estiveram em mais de uma categoria.

\section{Resultados das abordagens centradas no mindful eating na saúde mental}

Nessa categoria 13 artigos se enquadram, com pesquisas avaliando as repercussões de intervenção baseada no mindful eating que envolviam a saúde mental dos participantes buscaram avaliar os impactos: (1) nas escolhas alimentares, no comportamento alimentar e na alimentação emocional; (2) no consumo de doces em pacientes diabéticos; (3) no tratamento de transtornos alimentares e (4) no sofrimento psicológico.

Os estudos que tiveram entre seus objetivos avaliar melhorias no comportamento alimentar e melhorias nas escolhas alimentares (n=06; 30\%) trouxeram resultados satisfatórios, uma vez que foram observadas melhorias nos escores de compulsão alimentar, reduções dos níveis de alimentação emocional, melhorias nas escolhas alimentares e na ingestão de alimentos, diminuição da alimentação por razões externas, diminuição do consumo de alimentos com alta densidade calórica e com alto teor de gorduras, na ingestão energética e houve um aumento dos escores de comer consciente.

Em relação aos estudos envolvendo indivíduos portadores de diabetes $(n=02 ; 10 \%)$, após a intervenção foi observada redução significativa no consumo de doces. Para os estudos feitos com portadores de TA $(n=02 ; 10 \%)$ observou-se diminuição da restrição alimentar, melhora no sofrimento emocional e melhora nos escores do EAT-26 (usado para avaliar sintomas e comportamentos característicos de TA).

Nos estudos que avaliaram alguma característica emocional dos participantes $(n=03$; 15\%), foi observado redução nos níveis de estresse e de depressão, e melhora no sofrimento emocional após a participação nas intervenções.

\section{Resultados das abordagens centradas no mindful eating na saúde física}

Foram analisados 10 artigos nessa categoria, onde as pesquisas envolvendo a intervenção baseada no mindful eating que envolviam a saúde física dos participantes buscaram avaliar os impactos: (1) no peso, IMC e na manutenção do peso; (2) melhorias na glicemia de jejum, glicemia pós prandial e hemoglobina glicada e (3) níveis de proteína C reativa.

Os estudos que objetivaram avaliar melhorias em relação ao peso, IMC e manutenção do peso $(n=05 ; 25 \%)$ também apresentaram resultados promissores, uma vez que, observou-se que em 10,0\% (n= 02) houve manutenção do peso, e nos demais houve um aumento da eficácia para perda de peso, diminuição do peso, IMC e circunferência da cintura. No estudo desenvolvido com a condição clínica de anorexia nervosa 01 (5,0\%) houve um aumento do IMC.

Nos estudos que avaliaram a glicemia e a hemoglobina glicada $(n=02 ; 10 \%)$ também apresentaram resultados satisfatórios, uma vez que a intervenção promoveu diminuição significativa na glicemia de jejum, na glicemia pós prandial e na hemoglobina glicada. 0 único estudo que analisou os níveis de proteína $C$ reativa também encontrou redução em seus níveis após intervenção.

\section{Resultados das abordagens centradas no intuitive eating na saúde mental, emocional e física}

Os 04 estudos analisados envolvendo a intervenção baseada no intuitive eating que envolviam a saúde mental dos participantes buscaram avaliar os impactos: (1) no comportamento alimentar, alimentação emocional; (2) nos aspectos emocionais e (3) apreciação corporal.

Apesar de 01 estudo (5\%) ${ }^{19}$ não ter encontrado mudanças significativas para o comportamento alimentar e para parâmetros emocionais com a intervenção, os outros 02 $(10 \%)^{27,34}$ encontraram resultados positivos após intervenção, dentre eles: redução significativa da alimentação emocional e fome física, aumento da pontuação para apreciação 
corporal e melhora na mentalidade da dieta dos participantes, que iniciaram comportamentos de vida mais intuitivos e aumento da autoestima.

Apenas 01 (5\%) estudo avaliou o impacto da intervenção baseada no intuitive eating na saúde física dos participantes. 0 estudo de Anglin ${ }^{31}$ buscou avaliar os efeitos de uma abordagem centrada no IE $v s$. abordagem baseada restrição calórica (RC) na perda de peso, valores de IMC e de circunferência da cintura (CC) e concluiu que a perda de peso e a redução do IMC foram maiores para o grupo RC do que para o grupo IE. Em relação a CC não houve diferença significativa.

\section{DISCUSSÃO}

As 20 pesquisas que investigaram as repercussões das abordagens centradas no "mindful eating" e no "intuitive eating" na saúde de indivíduos adultos apontaram que, tais intervenções repercutiram de forma positiva tanto na saúde física, como mental dos sujeitos ${ }^{18-}$ 37.

O comportamento alimentar é delineado por uma intensa interação entre uma diversificada gama de fatores, como os fisiológicos, sociais, genéticos, psicológicos e culturais, tornando-o complexo, uma vez que o comer é um ato social que vai além das necessidades básicas de alimentação indispensáveis para a sobrevivência humana. No momento da alimentação o sujeito busca atender não apenas a sua necessidade fisiológica, mas também a sua necessidade hedônica (prazerosa) e emocional ${ }^{38}$.

Sob este prisma, as intervenções centradas no mindful eating e intuitive eating ganham destaque por reunirem e valorizarem os aspectos emocionais e psicossociais que perpassam a alimentação, além dos fisiológicos. Ambas as intervenções não possuem como foco mudar os alimentos que o indivíduo consome, mas sim entender qual a relação que os indivíduos possuem com a comida, e sobre como a mente e o corpo entendem a experiência do comer, diferente das abordagens tradicionais que são caracteristicamente prescritivas e restritivas, não levando em consideração os aspectos psicossociais do comer ${ }^{39}$.

Embora o comportamento e as práticas alimentares sejam influenciados por numerosos fatores, o fator emocional ganha destaque e é apontado como o principal determinante dos excessos alimentares. A adesão a um controle alimentar tende a ser dificultada pelas emoções, pois a função racional pode ser inibida quando a emocional se sobressai ${ }^{40}$.

Nesse sentido, os resultados promissores obtidos com o mindful eating e o intuitive eating guardam íntima relação com o olhar que ambas as abordagens trazem para o comer emocional, auxiliando o indivíduo tanto a identificar as diferenças entre fome física versus fome emocional, quanto a desenvolverem habilidades para lidar com emoções e sensações difíceis, evitando a busca pela comida como forma de auto-regulação emocional ${ }^{12}$.

A partir dessas intervenções, os sujeitos tem a possibilidade de desenvolver e treinar a habilidade de observar a sua própria experiência, sem julgamento ou críticas, o que pode evitar comportamentos alimentares disfuncionais, e também se instrumentalizar para lidar e aceitar os sentimentos negativos, sem, necessariamente, suprimi-los por meio da comida ${ }^{41}$.

0 entendimento dos aspectos psicossociais que perpassam a alimentação e participam da regulação do comportamento alimentar deve ser parte essencial das abordagens que tem a intenção de auxiliar os sujeitos a melhorar sua relação com a comida. Sob esta perspectiva, as intervenções baseadas no ME e IE ganham destaque por priorizarem a melhoria dessa relação, com intervenções que auxiliam o indivíduo a observar a sua própria experiência com o comer, sem julgamento ou críticas, e começar a aprender a manejar e aceitar os sentimentos negativos com mais acolhimento, gentileza e compaixão, minimizando o impulso de lidar com esses sentimentos por meio da comida ${ }^{41}$.

Um estudo investigou os benefícios de um grupo baseado em ME no tratamento de transtornos alimentares, e teve resultado positivo, pois após a intervenção foi observado 
melhora significativa nos escores do EAT-26 ${ }^{36}$. Esse resultado foi de encontro com uma revisão nacional, na qual a abordagem ME obteve resultados positivos 5 .

Há impactos positivos mentais que podem decorrer de programas que incorporam e valorizam os princípios do ME. Esses impactos na saúde mental podem ser justificados pelo fato das intervenções baseadas nos protocolos de ME ajudarem os indivíduos a lidar com fatores psicológicos que afetam os padrões alimentares, pois essa intervenção os ajuda a terem maiores percepções de seus pensamentos, emoções e sensações angustiantes e por consequência pararem de se alimentarem em resposta a sinais emocionais e começarem a se alimentarem em resposta a sinais físicos (fome e saciedade) ${ }^{19}$.

A prática de autocuidado que ambas as intervenções proporcionam pode ser importante para a promoção da apreciação corporal. Níveis elevados de apreciação corporal têm sido associados à menor frequência de conversas corporais negativas entre as mulheres, promovendo assim uma imagem corporal positiva. 0 conceito de imagem corporal positiva reflete a manutenção de atitudes favoráveis em relação ao corpo, cuidar do corpo, respeitar o corpo e proteger o corpo ao rejeitar o padrão de beleza corporal muitas vezes irreal imposto pela mídia como sendo sinônimo de beleza ${ }^{42}$.

Em relação aos resultados que tiveram desfechos relacionados ao peso, IMC e circunferência da cintura, os participantes mantiveram o peso, ou seja, não houve modificação do peso após a intervenção, apesar disto não devem ser vistos como desfechos negativos, visto que ambas as intervenções não possuem como foco a perda de peso. A perda de peso pode vir como consequência da melhoria na relação do sujeito com o comer e com a comida, bem como da maior habilidade de estar atento aos sinais internos e externos da alimentação. Uma revisão sistemática com metanálise analisou ensaios clínicos randomizados, e ao comparar os desfechos relacionados ao peso em estudos com ME vs. aqueles com programas de intervenções convencionais, mostrou que não houveram diferenças significativas ${ }^{10}$.

Os resultados positivos das variáveis bioquímicas também são justificados pela melhoria das escolhas alimentares. Os exercícios de yoga promovem a conexão do corpo com a mente, não só ocasionando um relaxamento mental e gerando redução do estresse físico e mental, mas também atua no mecanismo da atividade humoral e do sistema nervoso, portanto é útil para reduzir a glicose no sangue ${ }^{27}$.

Dos 20 estudos analisados apenas 01 mostrou resultado negativo, em que após a intervenção houve aumento nas pontuações relacionadas ao hábito indesejado de "beliscar" e redução nos níveis de autocompaixão, sem alteração para os níveis de $\mathrm{ME}^{20}$, com possível influencia das limitações, o que pode justificar o desfecho negativo da intervenção. Primeiramente a intervenção não foi feita por um instrutor, sendo realizada por email, o que pode ser uma das possíveis causas para o desfecho da redução dos níveis autocompaixão, bem como estes poderiam ter tido um nível elevado de autojulgamento, em relação aos seus hábitos.

Somado a isso, o desfecho do hábito indesejado de "beliscar" foi medido através de autorrelato (Self-Report Habit Index: SRHI) que pode capturar a experiência subjetiva do hábito e não os processos habituais reais em $\mathrm{si}^{20}$. Outro fator é que esses resultados podem indicar uma maior consciência desses hábitos através das práticas de ME e não o seu aumento. Em relação a não alteração dos níveis de $\mathrm{ME}$, o estudo relata que os participantes podem ter superestimado na primeira avaliação o seu nível de alimentação consciente, pois a percepção do comer sem consciência só é observado após ter contato co m a prática ${ }^{20}$.

\section{CONCLUSÃO}

Os resultados encontrados na presente revisão sugerem que a intervenção baseada em mindful eating é capaz de modificar as práticas alimentares dos indivíduos para práticas mais saudáveis e também modificar a relação que eles possuem com os alimentos. Somado a isso, juntamente com programas que abordam o intuitive eating, os resultados mostraram que este 
conjunto pode gerar impactos positivos na percepção que os indivíduos têm em relação aos seus próprios corpos, gerando um aumento de apreciação corporal.

Sugere-se então, que essas intervenções podem ser utilizadas para a melhoria da relação dos sujeitos com alimentação e questões relacionadas ao corpo, bem como dos sentimentos que perpassam essas questões, contribuindo com melhora da saúde física e mental dos indivíduos.

Como limitações do presente estudo, destaca-se que a presente revisão incluiu artigos publicados em 05 diferentes bases de dados, mas podem haver dados apresentados em outras fontes de divulgação científica que não foram alcançados. Outro ponto é que os resultados apresentados se referem apenas a estudos internacionais, o que limita a generalização dos achados para a população brasileira, uma vez que ainda não há estudos desenvolvidos e publicados nessa população. Por outro lado, os achados internacionais apresentados então, devem ser sinalizadores para a importância da temática no cenário nacional, bem como do próprio uso do mindful eating e do intuitive eating.

\section{REFERÊNCIAS}

1. Martinez S. A nutrição e a alimentação como pilares dos programas de promoção da saúde e qualidade de vida nas organizações. Mundo Saúde [Internet]. 2013 [citado em 10 jan 2020]; 37(2):201-7.

Disponível

em: https://www.revistamundodasaude.com.br/assets/artigos/2013/102/9.pdf DOI: http://dx.doi.org/10.15343/0104-7809.2013372201207

2. Rezende FAC, Penaforte FRO. Dieta e seus desfechos negativos em saúde. In: Rezende FAC, Penaforte FRO, Martins PC, organizadores. Comida, corpo e comportamento humano. São Paulo: IACI; 2020. p. 19-34.

3. Cavalcanti APR, Dias MR, Costa MJC. Psicologia e nutrição: predizendo a intenção comportamental de aderir a dietas de redução de peso entre obesos de baixa renda. Estud Psicol. (Natal) [Internet]. 2005 [citado em 10 jan 2020]; 10(1):121-9. Disponível em: https://www.scielo.br/j/epsic/a/gmrJkgnfrcSCXNjRG87rL3f/?lang=pt\&format=pdf DOI: https://doi.org/10.1590/S1413-294X2005000100014

4. Bays JC. Mindful eating- a guide to discovering a healthy and joyful relationship with food. Boston \& London: Shambhala; 2009. p. 10-232.

5. Almeida CC, Assumpção AA. A eficácia do mindful eating para transtornos alimentares e obesidade: revisão integrativa. Pretextos [Internet]. 2018 [citado em 10 jan 2020]; 3(6):25-36. Disponível

http://periodicos.pucminas.br/index.php/pretextos/article/view/18403/13607

6. Kristeller JL, Hallett CB. An exploratory study of a meditation-based intervention for binge eating disorder. J Health Psychol. [Internet]. 1999 [citado em 10 jan 2020]; 4(3):357-63. Disponível em: https://journals.sagepub.com/doi/10.1177/135910539900400305. DOI: http://dx.doi.org/10.1177/135910539900400305

7. Rossy L. The Mindfulness-Based Eating Solution: proven strategies to end overeating, satisfy your hunger, and savor your life. Oakland: New Harbinger; 2016. p. 5-216.

8. Job AR, Oliveira ACS. Percepção dos acadêmicos de nutrição sobre a efetividade a longo prazo dos métodos de dietas restritivas para a perda e o controle de peso. Rev Bras Obes Nutr Emagr. [Internet]. 2019 [citado em 10 jan 2020]; 13(78):291-8. Disponível em: http://www.rbone.com.br/index.php/rbone/article/view/941/671

9. Cadena-Schlam L, López-Guimerà G. Intuitive eating: An emerging approach to eating behavior. Nutr Hosp. [Internet]. 2015 [citado em 10 jan 2020]; 31(3):995-1002. Disponível em: https://scielo.isciii.es/pdf/nh/v31n3/01revision01.pdf.

http://dx.doi.org/10.3305/nh.2015.31.3.7980

10. Artiles RF, Staub K, Aldakak L, Eppenberger P, Ruhli F, Bender N. Mindful eating and common diet programs lower body weight similarly: systematic review and meta-analysis. Obes Rev. [Internet]. 2019 [citado em 10 jan 2020]; 20(11):1619-27. Disponível em: 
https://onlinelibrary.wiley.com/doi/epdf/10.1111/obr.12918.

DOI: https://doi.org/10.1111/obr.12918

11. Schaefer JT, Magnuson AB. A review of interventions that promote eating by internal cues. J Acad Nutr Diet. [Internet]. 2014 [citado em 10 jan 2020]; 114(5):734-60. Disponível em: https://linkinghub.elsevier.com/retrieve/pii/S2212267213018960. DOI: http://dx.doi.org/10.1016/j.jand.2013.12.024

12. Warren JM, Smith N, Ashwell M. A structured literature review on the role of mindfulness, mindful eating and intuitive eating in changing eating behaviours: effectiveness and associated potential mechanisms. Nutr Res Rev. [Internet]. 2017 [citado em 10 jan 2020]; 30(2):272-83. Disponível em: https://www.cambridge.org/core/journals/nutrition-researchreviews/article/structured-literature-review-on-the-role-of-mindfulness-mindful-eating-andintuitive-eating-in-changing-eating-behaviours-effectiveness-and-associated-potential-

mechanisms/351A3D01E43F49CC9794756BC950EFFC.

DOI: http://dx.doi.org/10.1017/S0954422417000154

13. O'Reilly GA, Cook L, Spruijt-Metz D, Black DS. Mindfulness-based interventions for obesity-related eating behaviours: a literature review. Obes Rev. [Internet]. 2014 [citado em 10 jan 2020]; 15(6):453-61. Disponível em: https://www.ncbi.nlm.nih.gov/pmc/articles/PMC4046117/. https://dx.doi.org/10.1111\%2Fobr.12156

14. Dunn C, Haubenreiser M, Johnson M, Nordby K, Aggarwal S, Myer S, et al. Mindfulness approaches and weight loss, weight maintenance, and weight regain. Curr Obes Rep. [Internet]. 2018 [citado em 10 jan 2020]; 7(1):37-49. Disponível em: https://ganepao.com.br/wp-content/uploads/2020/02/OK_Mindfulness-Approaches-andWeight-Loss-Weight-Maintenance.pdf. DOI: https://doi.org/10.1007/s13679-018-0299-6 15. World Health Organization. Constitution of the World Health Organization. Geneva: WHO; 1946.

16. Moher D, Liberati A, Tetzlaff J, Altman DG, The PRISMA Group. Preferred reporting items for systematic reviews and meta-analyses: the PRISMA Statement. Ann Intern Med. [Internet]. 2009 [citado em 10 jan 2020]; 151(4):264-9. Disponível em: http://www.prismastatement.org/documents/PRISMA-P\%20Statement\%20-

\%20Moher\%20Sys\%20Rev\%20Jan\%202015.pdf

17. Harris JD, Quatman E, Manring MM, Siston RA, Flanigan DC. How to write a systematic review. Am J Sports Med. [Internet]. 2014 [citado em 10 jan 2020]; 42(11):2761-8. Disponível em: https://journals.sagepub.com/doi/10.1177/0363546513497567. DOI: http://dx.doi.org/10.1177/0363546513497567

18. Lyzwinski LN, Caffery L, Bambling M, Edirippulige S. The mindfulness app trial for weight, weight-related behaviors, and stress in university students: randomized controlled trial. JMIR Mhealth Uhealth. [Internet]. 2019 [citado em 10 jan 2020]; 7(4):e12210. Disponível em: https://mhealth.jmir.org/2019/4/e12210/. DOI: https://doi.org/10.2196/12210

19. Gidugu V, Jacobs M.L. Empowering individuals with mental illness to develop healthy eating habits through mindful eating: results of a program evaluation. Psychol Health Med. [Internet]. 2019 [citado em 10 jan 2020]; 24 (2):177-86. Disponível em: https://pubmed.ncbi.nlm.nih.gov/30165751/. https://doi.org/10.1080/13548506.2018.1516295

20. Dibb-Smith A, Chapman J, Brindal E. Breaking habits with mindful snacking? An email-based intervention targeting unwanted snacking habits in an Australian sample. Eat Behav. [Internet]. 2019 [citado em 10 jan 2020]; 32:37-43. Disponível em: https://www.sciencedirect.com/science/article/abs/pii/S1471015318301818?via\%3Dihub. DOI: https://doi.org/10.1016/j.eatbeh.2018.11.006 
21. Webber KH, Mellin L, Mayes L, Mitrovic I, Saulnier M. Pilot Investigation of 2 nondiet approaches to improve weight and health. Altern Ther Health Med. [Internet]. 2018 [citado em 10 jan 2020]; 24(1):16-20. Disponível em: https://pubmed.ncbi.nlm.nih.gov/28646804/

22. Wnuk SM, Du CT, Van Exan J, Wallwork A, Warwick K, Tremblay L, et al. Mindfulness-Based eating and awareness training for post-bariatric surgery patients: a feasibility pilot study. Mindfulness [Internet]. 2018 [citado em 10 jan 2020]; 9(3):949-60. DOI: https://link.springer.com/article/10.1007/s12671-017-0834-7

23. Allirot X, Miragall M, Perdices I, Baños RM, Urdaneta E, Cebolla A. Effects of a brief mindful eating induction on food choices and energy intake: external eating and mindfulness state as moderators. Mindfulness [Internet]. 2017 [citado em 10 jan 2020]; 9(3):750-60. DOI: https://link.springer.com/article/10.1007/s12671-017-0812-0

24. Mason AE, Jhaveri K, Cohn M, Brewer JA. Testing a mobile mindful eating intervention targeting craving-related eating: feasibility and proof of concept. J Behav Med. [Internet]. 2017 [citado em 10 jan 2020]; 41(2):160-73. Disponível em: https://www.ncbi.nlm.nih.gov/pmc/articles/PMC5844778/. DOI: https://dx.doi.org/10.1007\%2Fs10865-017-9884-5

25. Mason AE, Epel ES, Kristeller J, Moran PJ, Dallman M, Lustig RH, et al. Effects of a mindfulness-based intervention on mindful eating, sweets consumption, and fasting glucose levels in obese adults: data from the SHINE randomized controlled trial. J Behav Med. [Internet]. 2016 [citado em 10 jan 2020]; 39(2):201-13. Disponível em: https://pubmed.ncbi.nlm.nih.gov/26563148/. DOI: 10.1007/s10865-015-9692-8

26. Stites SD, Singletary SB, Menasha A, Cooblall C, Hantula D, Axelrod S, et al. Pre-ordering lunch at work: results of the what to eat for lunch study. Appetite [Internet]. 2015 [citado em 10 jan 2020]; 84(1):88-97. Disponível https://www.sciencedirect.com/science/article/abs/pii/S0195666314004759. DOI: https://doi.org/10.1016/j.appet.2014.10.005

27. Youngwanichsetha $S$, Phumdoung S, Ingkathawornwong $T$. The effects of mindfulness eating and yoga exercise on blood sugar levels of pregnant women with gestational diabetes mellitus. Appl Nurs Res. [Internet]. 2014 [citado em 10 jan 2020]; 27(4):227-30. Disponível em: https://www.sciencedirect.com/science/article/pii/S0897189714000342?via\%3Dihub. DOI: http://dx.doi.org/10.1016/j.apnr.2014.02.002

28. Bush HE, Rossy L, Mintz LB, Schopp L. Eat for life: a work site feasibility study of a novel mindfulness-based intuitive eating intervention. Am J Health Promot. [Internet]. 2014 [citado em 10 jan 2020]; 28(6):380-8. Disponível em: https://journals.sagepub.com/doi/abs/10.4278/ajhp.120404-QUAN-186. DOI: https://doi.org/10.4278\%2Fajhp.120404-QUAN-186

29. Miller CK, Kristeller JL, Headings A, Nagaraja H, Miser WF. Comparative effectiveness of a mindful eating intervention to a diabetes self-management intervention among adults with type 2 diabetes: a pilot study. J Acad Nutr Diet. [Internet]. 2012 [citado em 10 jan 2020]; 112(11):1835-42. Disponível em: https://pubmed.ncbi.nlm.nih.gov/23102183/. DOI: 10.1016/j.jand.2012.07.036

30. Kidd LI, Graor CH, Murrock CJ. A mindful eating group intervention for obese women: a mixed methods feasibility study. Arch Psychiatr Nurs. [Internet]. 2013 [citado em 10 jan 2020]; 27(5):211-8. Disponível em: https://www.psychiatricnursing.org/article/S08839417(13)00072-1/fulltext. DOI: http://dx.doi.org/10.1016/j.apnu.2013.05.004

31. Anglin JC. Assessing the effectiveness of intuitive eating for weight loss - pilot study. Nutr Health [Internet]. 2012 [citado em 10 jan 2020]; 21(2):107-15. Disponível em: https://journals.sagepub.com/doi/10.1177/0260106012459994. DOI: https://doi.org/10.1177\%2F0260106012459994

32. Timmerman GM, Brown A. The effect of a mindful restaurant eating intervention on weight management in women. J Nutr Educ Behav. [Internet]. 2012 [citado em 10 jan 2020]; 44(1):22- 
8. Disponível em: https://linkinghub.elsevier.com/retrieve/pii/S1499404611002648. DOI: http://dx.doi.org/10.1016/j.jneb.2011.03.143

33. Miller CK, Kristeller JL, Headings A, Nagaraja H. Comparison of a mindful eating intervention to a diabetes self-management intervention among adults with type 2 diabetes: a randomized controlled trial. Health Educ Behav. [Internet]. 2014 [citado em 10 jan 2020]; 41(2):145-54. Disponível em: https://www.ncbi.nlm.nih.gov/pmc/articles/PMC4217158/. DOI: https://dx.doi.org/10.1177\%2F1090198113493092

34. Albers S. Using mindful eating to treat food restriction: a case study. Eat Dis. [Internet]. 2010 [citado em 10 jan 2020]; 19(1):97-107. Disponível em: https://pubmed.ncbi.nlm.nih.gov/21181582/. https://doi.org/10.1080/10640266.2011.533609

35. Cole RE, Horacek T. Effectiveness of the My Body Knows When Intuitive-Eating Pilot Program. Am J Health Behav. [Internet]. 2010 [citado em 10 jan 2020]; 34(3):286-97. Disponível em: https://pubmed.ncbi.nlm.nih.gov/20001186/. DOI: https://doi.org/10.5993/ajhb.34.3.4

36. Hepworth NS. A mindful eating group as an adjunct to individual treatment for eating disorders: a pilot study. Eat Dis. [Internet]. 2010 [citado em 10 jan 2020]; 19(1):6-16. Disponível em: https://www.tandfonline.com/doi/abs/10.1080/10640266.2011.533601. DOI: http://dx.doi.org/10.1080/10640266.2011.533601

37. Dalen J, Smith BW, Shelley BM, Sloan AL, Leahigh L, Begay D. Pilot study: Mindful Eating and Living (MEAL): weight, eating behavior, and psychological outcomes associated with a mindfulness-based intervention for people with obesity. Complement Ther Med. [Internet]. 2010 [citado em 10 jan 2020]; 18(6), 260-4. Disponível em: https://pubmed.ncbi.nlm.nih.gov/21130363/.

https://doi.org/10.1016/j.ctim.2010.09.008

38. Vaz DSS, Bennemann RM. Comportamento alimentar e hábito alimentar: uma revisão. UNINGÁ Rev. [Internet]. 2014 [citado em 10 jan 2020]; 20(1):108-12. Disponível em: http://revista.uninga.br/index.php/uningareviews/article/view/1557/1168

39. Palmeira L, Pinto-Gouveia J, Cunha M. Exploring the efficacy of an acceptance, mindfulness \& compassionate-based group intervention for women struggling with their weight (Kg-Free): a randomized controlled trial. Appetite [Internet]. 2017 [citado em 10 jan 2020]; 112(1):10716. Disponível em: https://pubmed.ncbi.nlm.nih.gov/28119138/. DOI: https://doi.org/10.1016/j.appet.2017.01.027

40. Magalhães P, Motta DG. Uma abordagem psicossocial do estado nutricional e do comportamento alimentar de estudantes de nutrição. Nutrire [Internet]. 2012 [citado em 10 jan 2020]; 37(2):118-32. Disponível em: http://sban.cloudpainel.com.br/files/revistas_publicacoes/359.pdf. DOI: http://dx.doi.org/10.4322/nutrire.2012.01

41. Silva BF, Martins ES. Mindful Eating na nutrição comportamental. Rev Cient Univiçosa [Internet]. 2017 [citado em 10 jan 2020]; 9(1):82-6. Disponível em: https://academico.univicosa.com.br/revista/index.php/RevistaSimpac/article/download/92 1/1029

42. Webb JB, Courtney BR, Etzel L, Padro MP. “Mom, quit fat talking- I'm trying to eat (mindfully) here!": evaluating a sociocultural model of family fat talk, positive body image, and mindful eating in college women. Appetite [Internet]. 2018 [citado em 10 jan 2020]; 126:169-75. Disponível

em: https://www.sciencedirect.com/science/article/abs/pii/S0195666317310279?via\%3Dihub. DOI: http://dx.doi.org/10.1016/j.appet.2018.04.003 
Editora Associada: Fernanda Carolina Camargo

\section{CONTRIBUIÇÕES}

Ana Flávia de Sousa Silva e Fernanda Rodrigues de Oliveira Penaforte contribuíram na concepção, análise e interpretação dos dados, redação e revisão. Camila Cremonezi Japur participou da coleta e análise dos dados, redação e revisão. João Henrique Fabiano Motarelli atuou na redação e revisão. Taisa Alves Silva e Thamara Smatiotto Buttros colaboraram na coleta e análise dos dados e revisão.

\section{Como citar este artigo (Vancouver)}

Silva AFS, Japur CC, Silva TA, Motarelli JHF, Buttros TS, Penaforte FRO. Repercussões das abordagens mindful eating e intuitive eating na saúde de indivíduos adultos: revisão integrativa. REFACS [Internet]. 2021 [citado em inserir dia, mês e ano de acesso]; 9(4):97188. Disponível em: inserir link de acesso. DOI: inserir link do DOI.

\section{Como citar este artigo (ABNT)}

SILVA, A. F. S.; JAPUR, C. C.; SILVA, T. A.; MOTARELLI, J. H. F.; BUTTROS, T. S.; PENAFORTE, F. R. O. Repercussões das abordagens mindful eating e intuitive eating na saúde de indivíduos adultos: revisão integrativa. REFACS, Uberaba, MG, v. 9, n. 4, p. 971-88, 2021. Disponível em: inserir link de acesso. Acesso em: inserir dia, mês e ano de acesso. DOI: inserir link do DOI.

\section{Como citar este artigo (APA)}

Silva, A.F.S., Japur, C.C., Silva, T.A., Motarelli, J.H.F., Buttros, T.S., \& Penaforte, F.R.O. (2021). Repercussões das abordagens mindful eating e intuitive eating na saúde de indivíduos adultos: revisão integrativa. REFACS, 9(4), 971-88. Recuperado em: inserir dia, mês e ano de acesso de inserir link de acesso. DOI: inserir link do DOI. 